\title{
Direct and Indirect Observation of Lithium in a Scanning Electron Microscope; Not Only on Pure Li!
}

\author{
P. Hovington ${ }^{1}$, M. Lagacé ${ }^{1}$, E. Principe ${ }^{2}$, S. Burgess ${ }^{3}$, A. Guerfi ${ }^{1}$, H. Demers ${ }^{4}$, R. Gauvin ${ }^{4}$, K. Zaghib ${ }^{1}$, \\ ${ }^{1 .}$ Hydro-Quebec Reaserch Institute, Varennes, Quebec, Canada \\ 2. Tescan - Orsay Holding, USA \\ 3. Oxford Instruments NanoAnalysis, High Wycombe UK. \\ 4. Department of Mining and Materials Engineering, McGill University, Montreal, Quebec, Canada.
}

Battery is one of the most used technologies in everyday life (cellular, electric vehicle or hybrid cars). Improvement in the specific capacity (energy by weight or volume) and charging rate has a potential to even significantly improve their used. Hence, the research in battery materials is very important, well founded and will have a direct economical and social impact. Most of the actual battery technology is based on the displacement of Lithium ion $\left(\mathrm{Li}^{+}\right)$from two active materials (i.e., graphite for the anode and $\mathrm{LiFePO}_{4}$ for the cathode). It is thus essential to determine the distribution and the amount of Li with a good spatial resolution $(<<1 \mu \mathrm{m})$ In terms of microstructural characterization $\mathrm{Li}$ is very difficult to analysed using conventional detector because it is a very light element and emits low energy x-rays $(52 \mathrm{eV})$. Optical Emission spectrometry and XPS can easily detect Li but without any no good lateral resolution. In counterpart, $\mathrm{Li}$ has a relatively high sputtered yield and low backscattered coefficient. Mass spectra have been used in dedicated secondary ion mass microscope (SIMS), either static or Time of Flight (TOF) but with, again, with a limited lateral resolution.

This work will present, for the first time, a scanning electron microscope coupled with a Ga ion beam that can easily detect Li using two different detectors; TOF-SIMS from collaborative work of Tescan and TOFWERK and Windowless energy dispersive spectrometer specially design for low noise and low energy works from Oxford Instrument NanoAnalysis.

We present in Figure 1 an ion-mass spectrum together with ion induced secondary electron image and Li-7 chemical mapping of $\mathrm{LiFePO} 4$ particles. In the mass spectra, taken with less than 30 seconds, we clearly see a very high $\mathrm{Li}$ peaks together with a low Fe peaks. We note the very sharps peaks of $\mathrm{Li}$ at the mass 7 (charge $=1$ ) with no convolution which is ideal for chemical identification and localisation. The Li mapping images with a field of view of $1.5 \mu \mathrm{m}$ clearly revealed the LiFePO4 particle with a spatial resolution much lower than $1 \mu \mathrm{m}$. One of the challenges with the TOF-SIMS will be to obtained quantitative results, especially on non planar surface. TOF-SIMS analysis on pre-flattened 'thick lamella' is now underway to minimize this effect. Optimization of this instrument and new results will be presented.

In addition to Li detection using the TOF-SIMS, we present, in Figure 2, Li K peaks not only from a Li standard, produced at Hydro-Quebec, but also on a $\mathrm{Li}_{x} \mathrm{~S}_{1-\mathrm{x}}$ polysulfide samples that are very important to understand the behavior of Li-S battery having a specific energy density 10x higher than standard Li-ion cathode material. To our knowledge it is the first time Li peaks is presented on a non pure Li film using EDS detector. For the Li films we clearly see a very high signal/noise Li peak and a good separation with the noise peak that is forming a small bump at the low end side of the $\mathrm{Li}$ peak. For the $\mathrm{Li}_{x} \mathrm{~S}_{1-\mathrm{x}}$ sample, the $\mathrm{Li} \mathrm{K}$ peak can be clearly distinguished from the noise peak and from the $\mathrm{S} \mathrm{Ll}$ peaks. Both peaks ( $\mathrm{Li} \mathrm{K}$ and $\left.\mathrm{S} \mathrm{L}_{1}\right)$ are found below $0.5 \mathrm{keV}$ so analysis at $1 \mathrm{kV}$ (or lower energy) at a very high spatial resolution can be made $(<30 \mathrm{~nm})$ in the SEM.

Li can also be indirectly observed using the variation of the BSE coefficient has a function of Li alloying in a Si anode. Figure 3 presents the variation of BSE as a function of composition for the Li-S complex. We can clearly see a decrease of $50 \%$ between $\mathrm{Si}$ and $\mathrm{Li}_{22} \mathrm{Si}_{5}$. This observation was clearly observed using in situ discharge of the $\mathrm{Si}$ anode particle (10x more energy than standard graphite anode) where some grains, or parts of grains, have greatly change their BSE yield with decreasing voltage.

In conclusion, the used of two detectors capable of detecting $\mathrm{Li}$ and not only using pure $\mathrm{Li}$ film, is promising for Li detection with a good spatial resolution on technological bulk sample and, we think, will be able to accelerate battery development at Hydro-Quebec and also in similar research centers. 


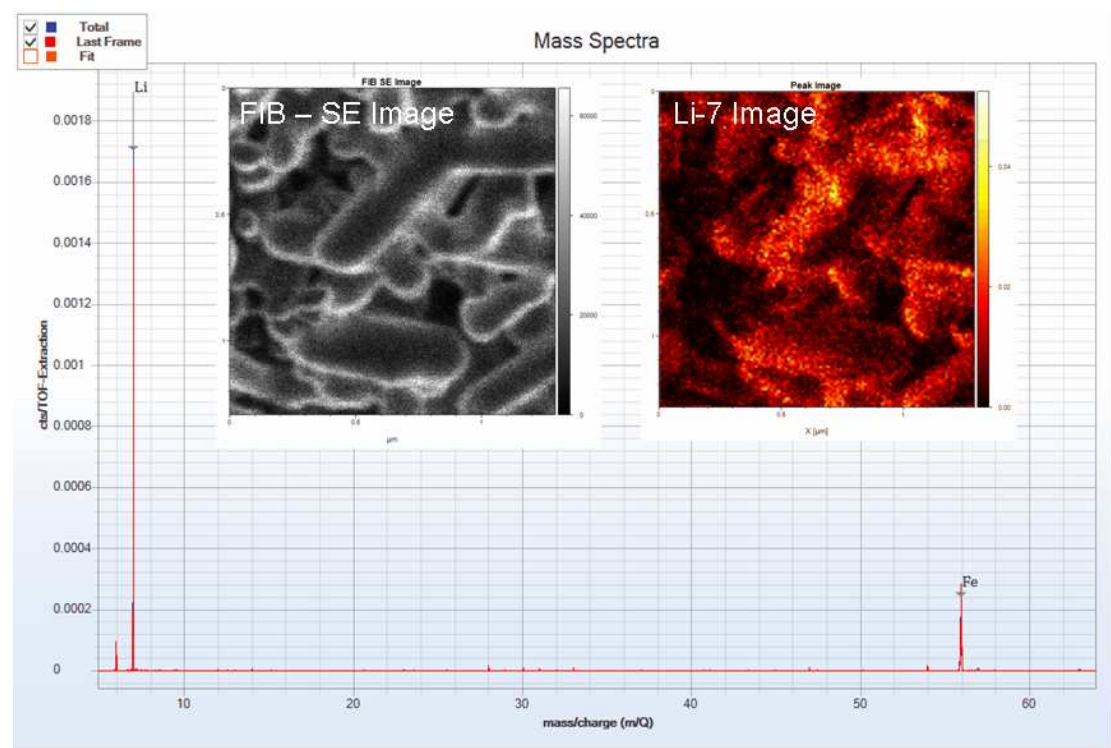

Figure 1. Mass Spectrum taken from a FIB scanning on $\mathrm{LiFePO}_{4}$ particles. Also shown in insert ion induced secondary electron image and $\mathrm{Li}$ image (field of view $1.5 \mu \mathrm{m}$ )

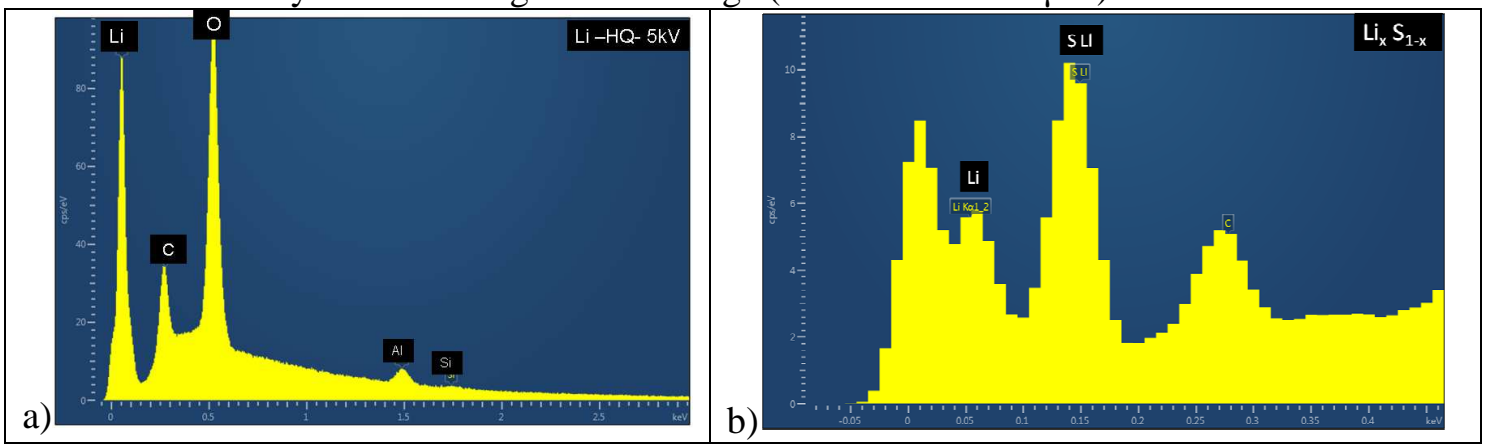

Figure 2. Li spectrum taken on a pure Li laminated films (produce at Hydro-Quebec) and (b) from a polysulfide $\left(\mathrm{Li}_{\mathrm{x}} \mathrm{Si}_{1-\mathrm{x}}\right)$.

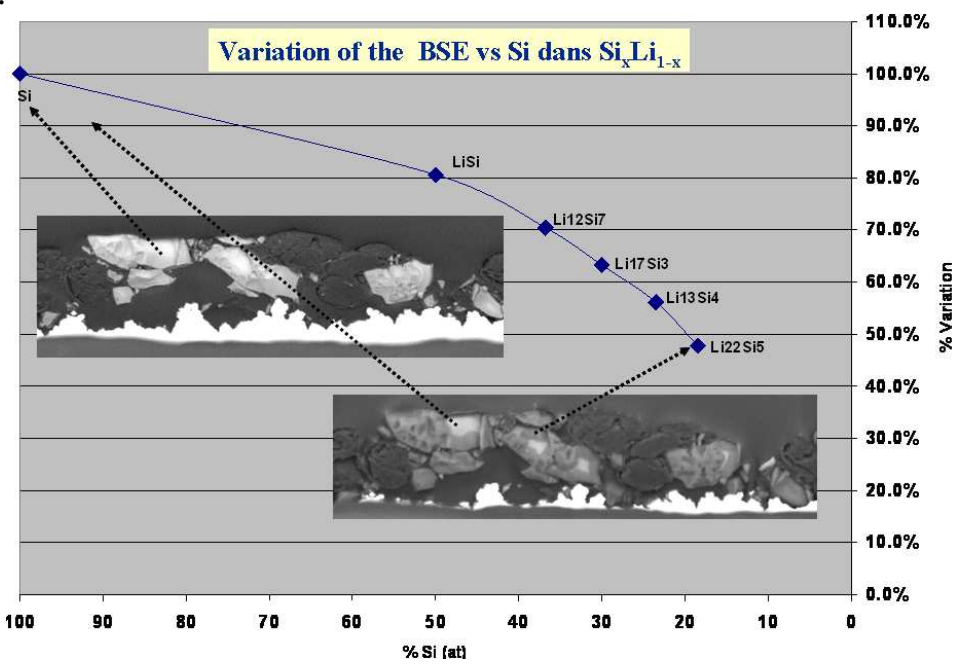

Figure 3. Variation of the Backscattered coefficient (BSE) vs. $\mathrm{Si}$ in the $\mathrm{Si}_{\mathrm{X}} \mathrm{Li}_{1-\mathrm{X}}$ complex. Insert is showing a cross-section of the electrode at the beginning and at the end of the discharge (white phase $=\mathrm{Si}$, gray phase $=\mathrm{Li}_{22} \mathrm{Si}_{5}$ ). Cycling is done in situ in the SEM. 